\title{
ERRATUM
}

\section{A Study on the Prevention of Salmonella Infection by Using the Aggregation Characteristics of Lactic Acid Bacteria} Min-Soo Kim, Yeo-Sang Yoon, Jae-Gu Seo, Hyun-Gi Lee, Myung-Jun Chung and Do-Young Yum R\&D Center, Cellbiotech Co. Ltd., Gyeonggi-do, Korea

http://dx.doi.org/10.5487/TR.2013.29.2.129

Toxicological Research Vol. 29, No. 2, pp. 129-135 (2013)

The authors regret that there is an error in the first sentence of the method section for 'LAB and S. typhimurium preparation'. The correct text is shown below in bold.

For the LAB, Lactobacillus acidophilus LA1 (KCTC 11906BP) that was used and orally administered once in a two day in this study was cultured from the CELLBIOTECH Co. Ltd. (Gimpo, Korea). 